\title{
ARTMAP Neural Network Classification of Land Use Change
}

\author{
G.A. Carpenter ${ }^{1}$, S. Gopal ${ }^{2}$, B. M. Shock ${ }^{3}$, and C. E. Woodcock ${ }^{4}$
}

\section{Abstract}

The ability to detect and monitor changes in land use is essential for assessment of the sustainability of development. In the next decade, NASA will gather high-resolution multi-spectral and multi-temporal data, which could be used for detecting and monitoring long-term changes. Existing methods are insufficient for detecting subtle long-term changes from high-dimensional data. This project employs neural network architectures as alternatives to conventional systems for classifying changes in the status of agricultural lands from a sequence of satellite images.

Landsat TM imagery of the Nile River delta provides a testbed for these land use change classification methods. A sequence of ten images was taken, at various times of year, from 1984 to 1993. Field data were collected during the summer of 1993 at 88 sites in the Nile Delta and surrounding desert areas. Ground truth data for 231 additional sites were determined by expert site assessment at the Boston University Center for Remote Sensing. The field observations are grouped into classes including urban, reduced productivity agriculture, agriculture in delta, desert/coast reclamation, wetland reclamation, and agriculture in desert/coast. Reclamation classes represent land use changes. A particular challenge posed by this database is the unequal representation of various land use categories: urban and agriculture in delta pixels comprise the vast majority of the ground truth data available in the database.

A new, two-step training data selection method was introduced to enable unbiased training of neural network systems on sites with unequal numbers of pixels. Data were successfully classified by using multidate feature vectors containing data from all of the available satellite images as inputs to the neural network system.

Keywords: ARTMAP, Neural network, Landsat TM, Land use change

\section{Introduction}

Traditional methods for land use classification using remotely sensed data require labor intensive analysis by experts. This paper presents a method that automates analysis of land use change data collected by satellite. The key requirements to apply this method are geographically registered multispectral satellite images

\footnotetext{
${ }^{1}$ Department of Cognitive and Neural Systems, Boston University, 677 Beacon St, Boston, MA 02215, USA, gail@cns.bu.edu.

${ }^{2}$ Center for Remote Sensing, Boston University, 675 Commonwealth Ave, Boston, MA 02215, USA, suchi@crsa.bu.edu.

${ }^{3}$ Department of Cognitive and Neural Systems, Boston University, 677 Beacon St, Boston, MA 02215, USA, bshock@cns.bu.edu

${ }^{4}$ Center for Remote Sensing, Boston University, 675 Commonwealth Ave, Boston, MA 02215, USA, curtis@crsa.bu.edu
} 
capturing land usage patterns and sample ground truth identifications that can be used to train the ARTMAP neural network system and verify its performance.

This study demonstrates the use of an ARTMAP neural network to identify land use changes in the Nile River delta and surrounding regions. The classifications made by the system enable users to visualize patterns of rural land use change. The methodology is general enough to be applicable to other land use change identification problems.

The traditional method of change detection described in Lenney et al. (1996) is a labor intensive technique requiring a great deal of hand crafting and expertise to create a system that can separate land use classes based on spectral characteristics. Using an ARTMAP neural network classifier automates some of this process of change detection, avoiding the need to hand craft a classifier for a specific problem. This ARTMAP methodology allows the identification of changes across a sequence of images of a given area. These images need not be taken under the same seasonal, atmospheric, or illumination conditions, and sensor calibration need not be consistent across the sequence. The ARTMAP system overcomes these inconsistencies by learning to identify the multidate spectral signatures of image pixels. This method is an extension of established single-date neural network landcover classification methods (Carpenter et al., 1997; Carpenter et al., 1999a; Carpenter et al., 1999b; Gopal et al., 1999). Data are classified by using multidate feature vectors containing data from all of the available satellite images as inputs to the neural network system.

\section{Data}

Ten Landsat TM images of the Nile River delta region and surrounding areas were taken at various times of year between 1984 and 1993. The images form the dataset used by Lenney et al. (Lenney et al., 1996) to study the classification of land use changes using multitemporal NDVI vegetation index features. The images were geometrically registered and normalized as described in that study.

Field data were collected during the summer of 1993 at 88 sites in the study area. Ground truth data from 231 additional sites were determined by expert image analysis at the Boston University Center for Remote Sensing. These data were combined to form a database of 319 sites. The database of was partitioned into four subsets, each containing between 89 and 91 sites. This allowed each of the four subsets to be used, in turn, as a test set to evaluate the performance of an ARTMAP classifier which has been developed and trained on the sites in the other three subsets. Carpenter et al. (1999b) describe the use of such a cross-validation method to evaluate machine learning systems while making full use of limited field data.

\section{Method}

\section{Data Preprocessing}

Prior to performing model selection, the data were preprocessed. This preparation consisted of computing transformations of the data and scaling all variables to the interval $[0,1]$. 
Dimensionality reduction was achieved by use of the Tasseled Cap transformation (Christ, 1985; Collins and Woodcock, 1996), which transforms six-band spectral data into three variables known as Brightness, Greenness, and Wetness. These account for most of the variation in Landsat TM images. The Brightness, Greenness, and Wetness values for each date were concatenated into a feature vector corresponding to each pixel. Additional features included, based on our experience with prior ARTMAP remote sensing applications (Carpenter et al., 1997; Carpenter et al., 1999b), were image pixel location coordinates and geographic zone designation indicator variables.

To conform to ARTMAP requirements, each input component was rescaled linearly to the range $[0,1]$ for all pixels in the dataset. The minimum value of each feature over the entire dataset was mapped to 0 and the maximum value of each feature was mapped to 1 .

\section{Model Selection}

It is necessary to establish what feature sets and parameters are likely to produce the most effective classifier system. The feature sets and parameters to be used for this application were determined by evaluation on the training sets. The feature set described above was consistently predicted to be the best of those considered.

Parameters varied across the four test systems. Details of the systems determined by this evaluation, including a table of parameters and complete Matlab code for the ARTMAP algorithm used, are available upon request from the authors.

\section{Training}

Each ARTMAP network was trained by presenting a random sequence of pixels from the training subset. A major challenge encountered with this database was that the available data from individual sites varied considerably. Sites ranged from a minimum of four pixels to a maximum of 3,440 pixels. It was important to ensure that each site was adequately represented in the neural network models while at the same time making use of as many data points as possible whenever more than four were available. This was achieved via a two-step pixel sampling process. The next training pixel was determined by first selecting a random training site and then selecting a random pixel from within that site. Training was continued until each site had been presented via representative pixels a number of times determined during the parameter selection phase.

\section{Testing of Selected Models}

Multiple trained ARTMAP networks were combined to form a committee voting system to improve the performance and stability of the classification. The number of networks to be combined $(V)$ was determined during parameter selection. Each voter was weighted equally. The committee system averaged the analog outputs $\sigma_{k}$ of the $V$ voters for each class $k$. A classification decision was made by selecting the class with maximum average output value:

$$
K^{\prime}=\underset{k}{\arg \max } \bar{\sigma}_{k}
$$


Combining two or more networks in a committee and making a classification decision on the basis of the average output of these committee members is a proven way of improving the expected performance of neural network systems (Bishop, 1995).

A similar scheme makes such a system an appropriate tool for site classifications. The analog values assigned to a single pixel by the voting system are estimates of its fuzzy membership in various classes. Averaging these values across all the pixels within a site gives average fuzzy membership estimates for the site. The class for which the site's fuzzy membership is maximal is the class to which the site is assigned by the system.

\section{Results and Conclusions}

The performance of the ARTMAP-based systems on this land use change classification dataset is encouraging. User's accuracy, the rate of correct classifications of sites in the ground truth database, averaged $84.6 \%$ for the four systems. Producer's accuracy, which weights classification rates by the estimated true proportions of land use change categories in the map, averaged $86.4 \%$. Each neural network attempts to optimize user's accuracy, having no knowledge of underlying class probabilities that might enable higher performance on producer's accuracy assessments.

A major advantage of using ARTMAP neural networks to generate land use change classification maps is that the confidence of classification decisions is readily available via the variables $\sigma_{k}$, which are the ARTMAP system's class probability estimates. In addition to a map showing land use changes, this methodology allows the user to generate a map of classification confidence. Identifying the areas in which the ARTMAP system's classifications are most likely to be incorrect could be used to guide manual editing of a land use change map to resolve differences between automatic classifications and expert judgments. These areas could even be used to guide future collection of ground truth data. For instance, there are large regions in the southwest quadrant of the study area that are classified by ARTMAP systems to be urban where there are no major urban settlements; Figure 2 shows that these coincide with the regions where the ARTMAP systems are least certain of their predictions.

For land use professionals, an attractive feature of ARTMAP neural network classifiers is that it automates a process that otherwise requires painstaking expert analysis. Once data collection has been performed, data can be analyzed rapidly and automatically. ARTMAP is a natural selection for rapid development of a land use change classification system. 


\section{References}

Bishop, C.M. 1995. Neural networks for pattern recognition. Oxford, Clarendon Press.

Carpenter, G.A., M.N. Gjaja, et al. 1997. ART neural networks for remote sensing: Vegetation classification from Landsat TM and terrain data. IEEE Transactions on Geoscience and Remote Sensing 35: 308-325.

Carpenter, G.A., S. Gopal, et al. 1999a. A neural network method for mixture estimation for vegetation mapping. Remote Sensing of Environment 70: 138-152.

Carpenter, G.A., S. Gopal, et al. 1999b. A neural network method for efficient vegetation mapping. Remote Sensing of Environment 70: 326-338.

Christ, E.P. 1985. A TM tasseled cap equivalent transformation for reflectance factor data. Remote Sensing of Environment 17: 301-306.

Collins, J.B. and C.E. Woodcock 1996. An assessment of several linear change detection techniques for mapping forest mortality using multitemporal Landsat TM data. Remote Sensing of Environment 56: 66-77.

Gopal, S., C.E. Woodcock, et al. 1999. Fuzzy neural network classification of global land cover from a 1 degrees AVHRR data set. Remote Sensing of Environment 67: 230-243.

Lenney, M.P., C.E. Woodcock, et al. 1996. The status of agricultural lands in Egypt: The use of multitemporal NDVI features derived from Landsat TM. Remote Sensing of Environment 56: 8-20.

\section{Tables}

\begin{tabular}{|c|c|c|c|c|c|}
\hline & $\begin{array}{c}\text { Partition } \\
1\end{array}$ & $\begin{array}{c}\text { Partition } \\
2\end{array}$ & $\begin{array}{c}\text { Partition } \\
3\end{array}$ & $\begin{array}{c}\text { Partition } \\
4\end{array}$ & $\begin{array}{c}\text { Average of } \\
\text { four } \\
\text { partitions }\end{array}$ \\
\hline $\begin{array}{c}\text { User's accuracy } \\
\text { (performance on } \\
\text { ground truth } \\
\text { dataset) }\end{array}$ & $89.9 \%$ & $85.4 \%$ & $84.3 \%$ & $79.1 \%$ & $\mathbf{8 4 . 6 \%}$ \\
\hline $\begin{array}{c}\text { Producer's accuracy } \\
\text { (weighted by map } \\
\text { percentages) }\end{array}$ & $88.5 \%$ & $86.9 \%$ & $90.2 \%$ & $80.1 \%$ & $\mathbf{8 6 . 4 \%}$ \\
\hline
\end{tabular}

Table 1. Performance (correct classification rate) of ARTMAP land use change classifier on four cross-validation partitions.

\section{Figures}




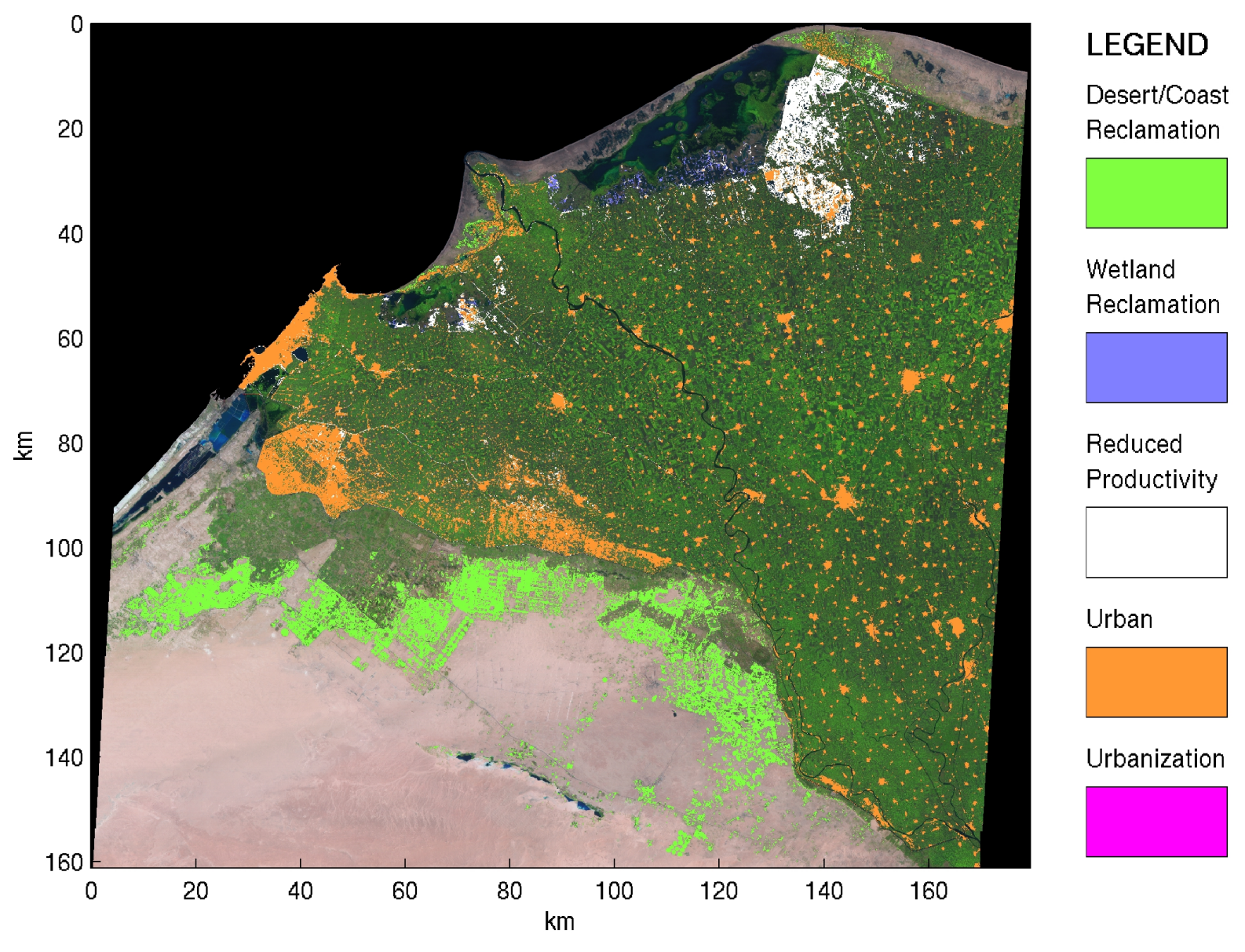

Figure 1. Composite map showing ARTMAP classifications of land use changes. Visible portions of the false color photograph indicate the classes agriculture in delta, agriculture in desert/coast, desert, and other.

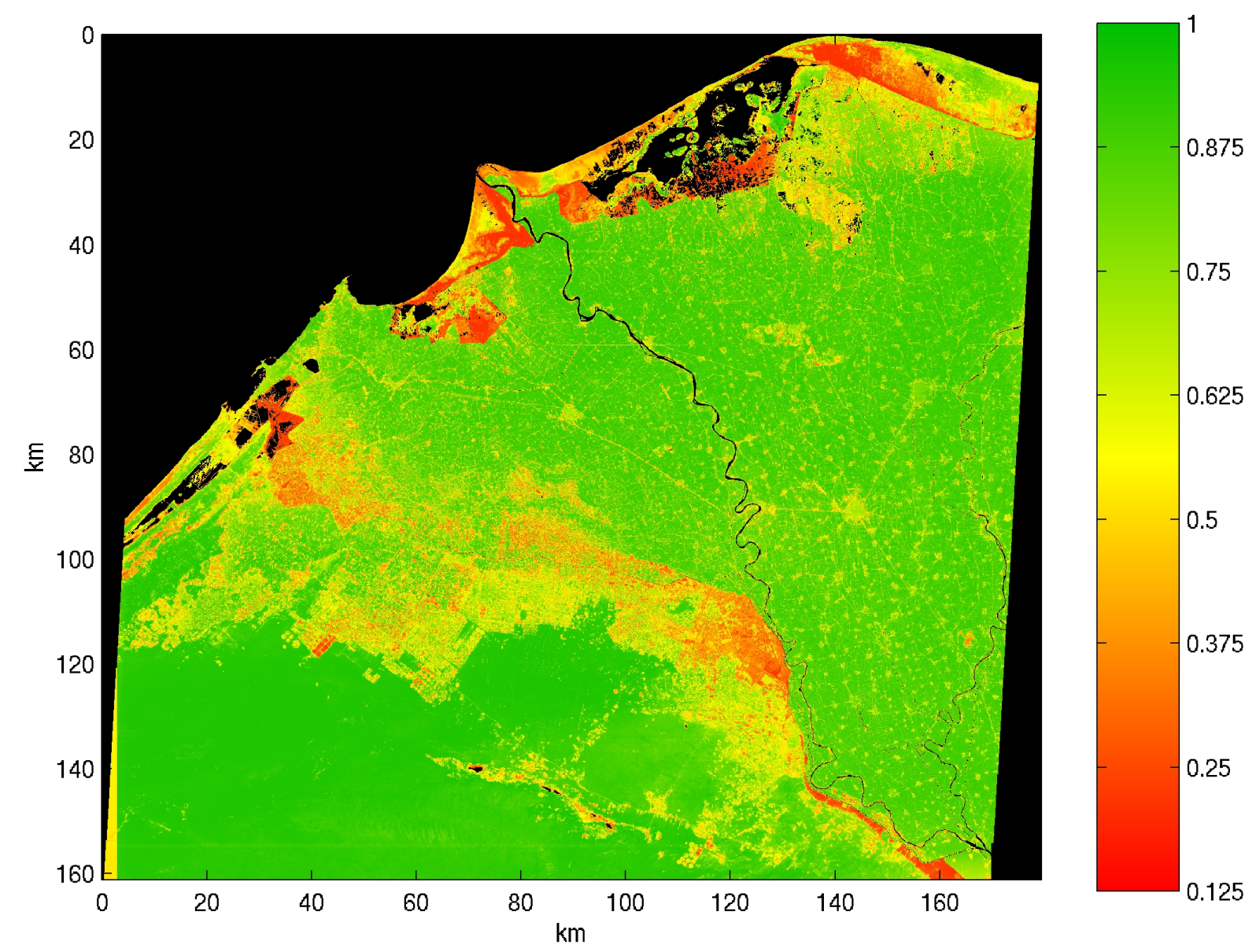

Figure 2. Map of ARTMAP system certainty estimates. The certainty is derived from internal ARTMAP parameters. Low values indicate that the system has difficulty distinguishing between two or more classes. 